\title{
Inflammatory cytokine expression following the use of bipolar electrocoagulation, ultracision harmonic scalpel and cold knife biopsy
}

\author{
PIETRO LITTA $^{1}$, CARLO SACCARDI ${ }^{1}$, SALVATORE GIZZO $^{1}$, LORENA CONTE ${ }^{1}$, \\ GIULIA AMBROSI $^{2}$, CLAUDIA SISSI ${ }^{2}$ and MANLIO PALUMBO ${ }^{2}$
}

\author{
${ }^{1}$ Department of Women's and Children's Health, Obstetrics and Gynecology Clinic, University of Padua; \\ ${ }^{2}$ Department of Pharmaceutical and Pharmacological Sciences, University of Padua, Padua 35128, Italy
}

Received March 29, 2014; Accepted January 21, 2015

DOI: $10.3892 / \mathrm{mmr} .2015 .3677$

\begin{abstract}
Electrical surgical devices may determine tissue damage through lateral thermal spread and activation of inflammatory processes. Several tissue effects are associated with the use of different surgical instruments. The aim of the present study was to compare tissue damage following the application of cold knife biopsy, bipolar electrocoagulation and the ultracision harmonic scalpel, through the analysis of inflammatory gene mediator expression. Three fragments of the round ligament (length $0.5 \mathrm{~cm}$ ) were obtained from 22 females who had undergone total or subtotal laparoscopic hysterectomy using three different modes of resection: Cold knife biopsy, bipolar electrocoagulation and ultracision harmonic scalpel. The tissue fragments were examined by quantitative polymerase chain reaction (qPCR) analysis of selected cytokines. Gene expression analysis demonstrated large standard deviations due to individual variability among patients and indicated variability in the concentrations of cytokines in the three different samples. The quantity of cytokine mRNA in the cold knife biopsy samples was generally greater than those obtained by other techniques. Tumor necrosis factor- $\alpha$ expression was significantly higher in the sample obtained with the ultracision harmonic scalpel and bipolar electrocoagulation $(\mathrm{P}=0.033)$ when compared with cold knife biopsy. The inflammatory response was analyzed by the quantification of gene expression through the use of qPCR. The ultracision harmonic scalpel and bipolar electrocoagulation triggered the inflammatory cascade and resulted in an increased production of cytokines compared with cold knife biopsy.
\end{abstract}

Correspondence to: Professor Salvatore Gizzo, Department of Women's and Children's Health, Obstetrics and Gynecology Clinic, University of Padua, 3 Giustiniani Street, Padua 35128, Italy

E-mail: ginecologia_padova@libero.it

Key words: inflammatory cytokine, tumor necrosis factor- $\alpha$, surgical thermal damage, quantitative polymerase chain reaction, ultrasonic scalpel device, bipolar electrocoagulation

\section{Introduction}

The electrical devices used during surgery for dissection and hemostasis may cause a local temperature increase, which eventually produces injury to the surrounding tissues by a process of thermal diffusion. Heating of a tissue has different effects depending on the temperature reached. Edema and activation of inflammatory processes occur at $34-44^{\circ} \mathrm{C}$, shrinkage of collagen structure occurs at $\sim 50-80^{\circ} \mathrm{C}$ and cell wall rupture and carbonization occur at temperatures $>100^{\circ} \mathrm{C}$ (1). The degree of lateral thermal spread and damage depends on the type of instrument used, electrical current intensity, time, electrode size and tissue conductivity $(2,3)$. A previous study demonstrated that monopolar diathermy generates the highest temperature and thus results in marked lateral thermal spread (4). Ultrasonic dissection was developed in the late 90 's for endoscopic surgery to reduce the risk of electrosurgery-induced tissue damage and in particular thermal complications associated with the use of monopolar electrodes (5). Lateral thermal spread is expected to be responsible for thermal damage. To evaluate lateral thermal damage, various methods have been proposed in previous studies, however, they often require demanding technical effort and long execution times $(6,7)$. In particular, ultrasound, magnetic resonance, infrared camera, thermal imaging and histology techniques have been employed (6,7). Currently, no fast and accurate laboratory method is available to appropriately quantify the damage associated with the use of electrical instruments in surgery. Clearly, analytical procedures must be adapted to instrument type, nature and composition of the tissue to be analyzed. As a consequence, a deep understanding of the physiological processes that are altered according to the different surgical procedures is difficult. The aim of the present study was to compare the effects of bipolar diathermy and ultrasonic dissection in the surgical site, by employing a simple analytical procedure. The present study focused on one of the most relevant biochemical consequences of tissue damage: The activation of the inflammatory cascade and the production of inflammatory mediators, in particular tumor necrosis factor (TNF)- $\alpha$, interleukin (IL)-1 and IL-6, produced by lymphocytes, macrophages, fibroblasts and endothelial cells. As an analytical technique, quantitative polymerase chain reaction 
(qPCR) was applied, which provides a rapid high throughput method to monitor protein expression in cells or tissues. RNA from clinical samples can be easily isolated and the mRNAs of a selected protein can be quantified by amplification of its corresponding cDNA. Thus, the present study quantitatively determined cytokine production using, as tissue samples, fragments of the round ligament obtained from a homogeneous group of patients undergoing laparoscopic hysterectomy with different scalpels.

\section{Materials and methods}

Tissue samples. In total, 22 females who had undergone total or subtotal laparoscopic hysterectomy at the Department of Women's and Children's Health, Obstetrics and Gynecology Clinic, University of Padua (Padua, Italy) were prospectively selected. Patients with benign gynecological conditions, aged between 18 and 50 years were included. Patients with a history of malignancy, pelvic endometriosis (8), endocrine pathology (9), preoperative use (within 2 months from surgery) of anti-inflammatory drugs, corticosteroids, gonadotropin-releasing hormone analogues, hormonal drugs (10) and immunosuppressive agents were excluded from the present study. All cases of preoperative/intraoperative pelvic inflammatory disease were also excluded (11). All subjects provided written informed consent previously approved by the local ethics committee. The present study was approved by the Institutional Review Board of the Ethics Committee of University of Padua.

During laparoscopic hysterectomy, performed by the same surgeon, three fragments of the round ligament (length $0.5 \mathrm{~cm}$ ) were obtained from each of the 22 patients. The three fragments corresponded to three different modes of resection: Cold knife biopsy, bipolar electrocoagulation and ultrasonic harmonic scalpel. Anthropometric parameters and blood cell count were recorded prior to surgery. Alterations in leukocyte concentration were monitored. Sampling was performed at the beginning of all interventions. The following surgical procedure was performed as previously described (12). The time of application of electrical current or ultrasonic device was always equal to 4-6 sec and the setting of devices was $50 \mathrm{~W}$ for bipolar forceps and level 5 for harmonic scalpel. Samples were frozen at $-80^{\circ} \mathrm{C}$ and subsequently subjected to quantitative mRNA determination. The tissue fragment was analyzed by qPCR and specific cytokines were quantitatively determined (Table I).

RNA extraction and reverse transcription. RNA extraction was performed using the SV total RNA Isolation kit provided by Promega (Madison, WI, USA). To begin the procedure, tissue samples were cut into small pieces with a sterile razor blade, weighed and suspended in the correct quantity of RNA lysis buffer (4 M GTC; $0.01 \mathrm{M}$ tris, $\mathrm{pH} 7.5 ; 0.97 \%$ $\beta$-mercaptoethanol) with the addition of $\beta$-mercaptohetanol. The round ligament tissue was then treated with a small homogenizer until no visible tissue fragments remained. From this lysate, RNA extraction was performed according to the manufacturer's instructions.

The RNA concentration in the purified extract was determined by spectrophotometric UV absorption measurements at
260 nm using a Nanodrop 1000 spectrophotometer (NanoDrop Technologies, Wilmington, DE, USA).

For each sample, $1 \mathrm{mg}$ of RNA was reverse transcribed into first-strand cDNA using the Transcriptor First Strand cDNA Synthesis kit (Roche Diagnostics, Indianapolis, IN, USA).

$q P C R$. Relative target protein mRNA quantification was evaluated by qPCR performed in a Roche LightCycler 1.5 (Roche Diagnostics) using a Light Cycler Fast Start DNA Master ${ }^{\text {PLus }}$ SYBR Green I kit (Roche Diagnostics). To normalize the data from different ligament samples, glyceraldehyde-3-phosphate dehydrogenase (GAPDH) transcripts were included as an internal control. For each reaction sample, $24 \mathrm{ng}$ of cDNA was used as a template and correct primer pairs (see Table I) were included at a final concentration of $50 \mathrm{nM}$. They were first denatured at $95^{\circ} \mathrm{C}$ for $10 \mathrm{~min}$ and then subjected to 45 cycles of amplification $\left(95^{\circ} \mathrm{C}\right.$ for $10 \mathrm{sec}, 60^{\circ} \mathrm{C}$ for $5 \mathrm{sec}$ and $72^{\circ} \mathrm{C}$ for $8 \mathrm{sec})$. The quality of the amplified products was checked by monitoring their melting profile. This was performed by continuously recording the SYBR Green I fluorescence signal while increasing the system temperature at a constant rate of $0.1^{\circ} \mathrm{C} / \mathrm{s}$.

For each reverse transcription (RT)-qPCR reaction, software analysis (LightCycler software version 3.5; Roche Diagnostics) provided Cp (crossing point), which is defined as the cycle number at which the fluorescence signal starts to exponentially increase. $\mathrm{Cp}$ were determined according to the second derivative maximum method (13).

Statistical analysis. Statistical analysis was performed using SPSS for Windows, version 19.0 (SPSS, Inc., Chicago, IL, USA). For comparison between groups analysis of variance was used. $\mathrm{P}<0.05$ was considered to indicate a statistically significant difference.

\section{Results}

Patient characteristics. The mean age of the patients was $46.63 \pm 5.33$ years. Eight $(36.4 \%)$ patients were nulliparous and $14(63.6 \%)$ were multiparous. None of the patients had relevant comorbidities. Four (18.1\%) patients presented hypertension. The mean body mass index of the patients was 23 (range 19-27). Twelve $(90.9 \%)$ patients were hospitalized due to the appearance of menometrorrhagia, which was unresponsive to medical treatment and eight (36.4\%) of these patients had an increase in uterine size due to fibromatosis, responsible for abdominal compression symptoms. In addition, two (9\%) patients underwent surgery for endometrial hyperplasia. Ten (45.5\%) patients underwent laparoscopic subtotal hysterectomy and twelve (54.5\%) underwent total hysterectomy. All pre-operative blood count analyses were normal. Round ligament sampling was successful in all patients. All of these samples were treated to extract total RNA. In the samples corresponding to three patients it was not possible to extract a sufficient quantity of RNA to perform gene expression analysis, which was likely due to problems with sample storage.

Gene expression. To evaluate alterations in inflammation-associated protein production according to the applied experimental procedure, relative quantitative evaluation of 
Table I. Sequence of the primers used to quantify inflammatory protein expression.

\begin{tabular}{lllr} 
Protein & \multicolumn{1}{c}{ Forward } & \multicolumn{1}{c}{ Reverse } & $\begin{array}{r}\text { Ampl } \\
\text { (bp) }\end{array}$ \\
\hline GAPDH & 5'-GCACCGTCAAGGCTGAGAAC-3' & 5'-ATGGTGGTGAAGACGCCAGT-3' & 69 \\
IL-1 & 5'-CCAGGGACAGGATATGGAGCA-3' & 5'-TTCAACACGCAGGACAGGTACAG-3' & 129 \\
IL-1R & 5'-TTTAAGCAGAAACTACCCGTTGCAG-3' & 5'-TCACGATGAGCCTATCTTTGACTCC-3' & 169 \\
IL-6 & 5'-AAGCCAGAGCTGTGCAGATGAGTA-3' & 5'-TGTCCTGCAGCCACTGGTTC-3' & 150 \\
IL-6R & 5'-GAGGGCTTCTGCCATTTCTGAG-3' & 5'-CCAGGTTCAGCTGACAACAAACA-3' & 69 \\
TNF- $\alpha$ & 5'-AGTATCCATGCTCTTGACCTTGTAG-3' & 5'-CCCGTAATTGCTCCAATCTG-3' & 139 \\
TNFR & 5'-GCCTGGAGTGCACGAAGTTG-3' & 5'-TCCACCGTTGGTAGCGATACATTA-3' & 156 \\
IL-8 & 5'-GACTTCCAAGCTGGCCGTG-3' & 5'-CTCCTTGGCAAAACTGCACC-3' & 81 \\
IL-8R & 5'-GAAGCACCATCATTCCCGTTG-3' & 5'-GACACAGCTTCAGCCCTGTTCTC-3' & 118 \\
IL-11 & 5'-CATCTAGGCCTGGGCAGGAA-3' & 5'-TCAGACAAATCGCCCTCAAGTG-3' & 179 \\
IL-11R & 5'-AGACCCTGGATGGTGCACTTG-3' & 5'-AGAAGTTCTCATAGTCGGCTGCTTG-3' & 102 \\
COX2 & 5'-GCCCTTCCTCCTGTGCCT-3' & 5'-AATCAGGAAGCTGCTTTTTAC-3' & 70 \\
iNOS & 5'-TGGAAGCGGTAACAAAGGAGA-3' & 5'-TGCTTGGTGGCGAAGATGA-3' & 79 \\
IL-10 & 5'-CCTGGAGGAGGTGATGCCCCA-3' & 5'-ATCGATGACAGCGCCGTAGCC-3' & 116 \\
\hline
\end{tabular}

Size of the resulting amplified fragment (Ampl) in base pairs is shown. IL, interleukin; TNF- $\alpha$, tumor necrosis factor- $\alpha$; R, receptor; COX, cyclooxygenase; iNOS, inducible nitric oxide synthase; GAPDH, glyceraldehyde-3-phosphate dehydrogenase.

A

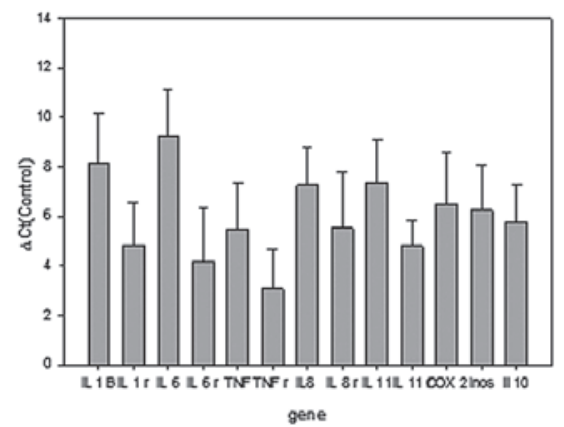

B

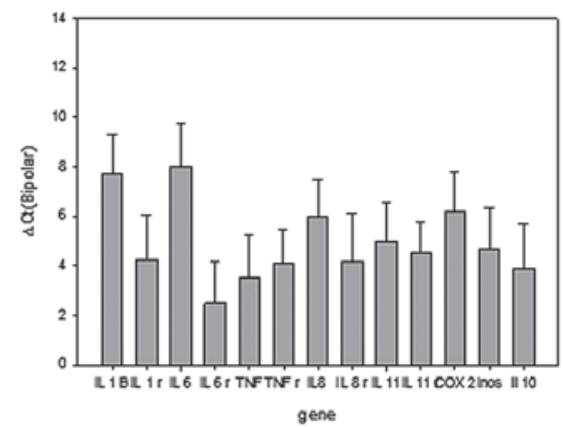

C

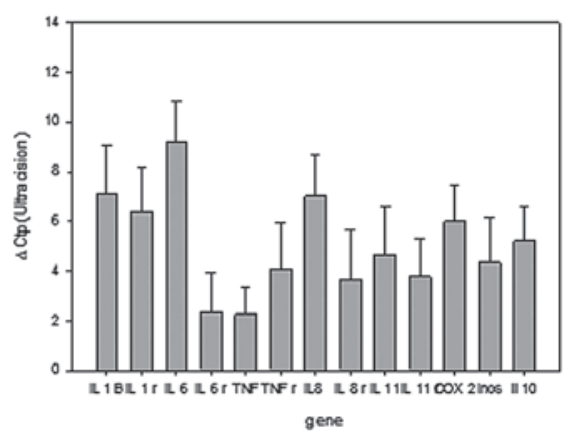

Figure 1. Alterations in gene expression compared with the control gene GAPDH $(\triangle \mathrm{Cp}$ ) for the samples from the round ligament obtained using (A) cold knife biopsy, (B) bipolar forceps and (C) the ultracision harmonic scalpel. IL, interleukin; TNF, tumor necrosis factor; COX, cyclooxygenase; INOS, inducible nitric oxide synthase; $\mathrm{R}$, receptor.

gene expression was performed using the housekeeping gene GAPDH as an endogenous control gene. Additionally, the efficiency of target amplification reaction (E) was comparable for the control and all target sequences as confirmed by the dilution method ( $\mathrm{E}=1.80-1.82$, data not shown).

The alterations in gene expression compared with the control gene GAPDH $(\triangle \mathrm{Cp})$ found for the samples of the round ligament obtained using cold knife (control), bipolar forceps and harmonic scalpel procedures are shown in Fig. 1. The results, which correspond to the average values obtained for each gene expression in all the examined patients, exhibit large standard deviations due to individual variability of gene expression. However, it is useful to underline that in the series of tissue obtained by cold knife biopsy (Fig. 1A) the variability of results is reduced if compared with the other two series. Additionally, on average, $\Delta \mathrm{Cp}$ values determined in the samples obtained by bipolar diathermy or ultracision harmonic scalpel are generally greater than those found in tissues recovered by cold knife biopsy. This is indicative of a general overexpression of inflammatory mediators in tissues treated with the ultracision technique or bipolar forceps as compared with cold knife biopsy. However, statistical analysis demonstrated that only TNF- $\alpha$ is significantly different among the three sampling conditions and that the corresponding mRNA concentration is lower in tissue cut with the harmonic scalpel and bipolar forceps $(\mathrm{P}=0.033)$. The data are shown in Table II.

Correlation between bipolar electrocoagulation and ultracision harmonic scalpel. In order to compare the results relative to bipolar electrocoagulation and ultracision harmonic scalpel, samples obtained by the use of the cold knife technique were considered as a further control sample, which allowed us to apply the $2^{-\Delta \Delta \mathrm{Cp}}$ method (14). Using the ratio 
Table II. $\Delta \mathrm{Cp}$ of inflammatory gene expression in round ligament fragments.

\begin{tabular}{lccc}
\hline Mediator & Cold knife biopsy & Bipolar electrocoagulation & Ultracision harmonic scalpel \\
\hline IL-1 $\beta$ & $8.14 \pm 4.13$ & $7.14 \pm 3.78$ & $7.73 \pm 3.17$ \\
IL-1R & $4.85 \pm 3.46$ & $4.49 \pm 3.15$ & $6.43 \pm 3.58$ \\
IL-6 & $9.24 \pm 3.74$ & $8.18 \pm 2.74$ & $9.20 \pm 3.33$ \\
IL-6R & $4.56 \pm 3.74$ & $2.96 \pm 2.82$ & $2.82 \pm 2.77$ \\
TNF- $\alpha$ & $5.53 \pm 3.62$ & $3.75 \pm 3.12$ & $2.46 \pm 1.86$ \\
TNFR & $3.15 \pm 2.68$ & $4.10 \pm 2.75$ & $4.20 \pm 4.07$ \\
IL-8 & $5.97 \pm 3.01$ & $7.02 \pm 3.36$ & $7.27 \pm 3.04$ \\
IL-8R & $6.00 \pm 3.40$ & $4.35 \pm 3.64$ & $4.10 \pm 3.36$ \\
IL-11 & $7.38 \pm 3.50$ & $4.99 \pm 3.13$ & $5.02 \pm 3.26$ \\
IL-11R & $4.80 \pm 2.12$ & $4.55 \pm 2.38$ & $4.08 \pm 2.46$ \\
COX2 & $6.75 \pm 3.64$ & $6.23 \pm 3.15$ & $6.04 \pm 5.71$ \\
iNOS & $6.27 \pm 3.59$ & $4.72 \pm 2.37$ & $4.74 \pm 2.97$ \\
IL-10 & $5.78 \pm 2.94$ & $4.11 \pm 3.29$ & $5.24 \pm 2.76$ \\
\hline
\end{tabular}

Data are expressed as the mean \pm standard deviation. IL, interleukin; TNF- $\alpha$, tumor necrosis factor- $\alpha$; R, receptor; COX, cyclooxygenase; iNOS, inducible nitric oxide synthase; GAPDH, glyceraldehyde-3-phosphate dehydrogenase.

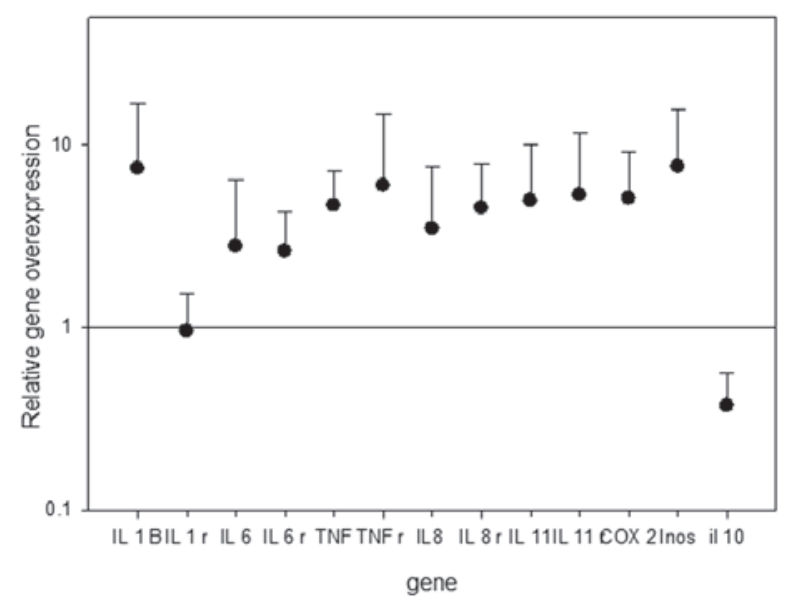

Figure 2. Relative gene overexpression following use of the ultracision harmonic scalpel compared with bipolar electrocoagulation. IL, interleukin; TNF, tumor necrosis factor; COX, cyclooxygenase; iNOS, inducible nitric oxide synthase; $\mathrm{R}$, receptor.

$2^{-\Delta \Delta \mathrm{Ct}}$ for ultracision $/ 2^{-\Delta \Delta \mathrm{Ct}}$ for bipolar cutting, the relative gene overexpression caused by one technique over the other can be easily visualized. As shown in Fig. 2, ultracision causes a partially higher production of cytokines compared with bipolar electrocoagulation, which is particularly evident for IL-1, TNF, IL-8, IL-11 and their respective receptors, as well as for inducible nitric oxide synthase and cyclooxygenase-2. However, this difference was not statistically significant (data not shown). This clearly reflects the high individual variability and the small number of analyzed patients.

\section{Discussion}

As reported by Harrell et al in 2004 (1), the use of electrical tools was introduced in surgery in 1926, and is currently part of common surgical practice. However, this raises concerns predominantly associated with the possible occurrence of complications caused by lateral thermal spread and scattering power. The recent development of minimally invasive surgical procedures has significantly stimulated research towards new instrumental techniques able to improve dissection and hemostasis events and to allow improved post-operative outcome. Thus, on one hand, bipolar electrodes have been made more efficient and on the other, new devices have been produced, which are based on alternative energy sources, including ultrasound (harmonic scalpel). Previous studies have indicated that the ultracision harmonic scalpel reduces blood loss, operative time and hospitalization time $(15,16)$. Additionally, due to its versatility, it can be effectively used in interventions that require good surgical practice, including cancer staging (17), minimal tissue trauma (when intraoperative frozen section analysis is necessary) $(18,19)$ and hemostasis, including during laparoscopic myomectomy (16). The available data on the extent of thermal damage following ultrasound or electricity application, have demonstrated that thermal damage caused by the ultracision harmonic scalpel is generally lower than bipolar diathermy, however, the variables affecting damage remain extremely abundant (20). The temperature recorded at the tips of the harmonic scalpel is slightly greater than in bipolar diathermy, however, the temperatures decreases more rapidly in the harmonic scalpel tips compared with the bipolar ones (4). In addition, the degree of damage varied with power setting. Perko et al (21) demonstrated that microscopic damage is more extensive when harmonic scalpel setting power 3 is applied for $>10 \mathrm{sec}$ and, for the same application time, when applied without interruption. For this reason, certain surgeons do not routinely use the harmonic scalpel setting to the power 5 . The present study is based on the knowledge that surgery damage induces a series of hormonal, metabolic and inflammatory alterations proportional to the severity of tissue trauma (22). An objective assessment of 
perioperative stress may be performed by measuring appropriate biochemical markers. The inflammatory response is greater following open surgery than laparoscopic surgery (23) and is significantly increased during the two days following surgery, as indicated by the high levels of IL-6 detected (24). As reported by Sietses et al (25) data from animal models on postoperative septic complications and tumor growth have demonstrated that preservation of immunological function, as occurring in the laparoscopic approach, may have significant clinical benefits. The inflammatory response not only triggers immunological mechanisms, but is also one of the most important causes of postoperative pain in patients who have undergone laparotomy surgery (26). The evaluation of inflammatory responses associated with the use of different surgical devices may assist in supporting minimally invasive surgery and understanding the mechanisms involved in postoperative pain. In the current literature, analysis of inflammatory mediators has been performed on peripheral blood (27). A study by Holub et al (22) demonstrated an increase in C-reactive protein, white blood cells, creatine kinase and IL-6 following laparoscopy, but did not highlight a significant difference between monopolar and bipolar electrosurgery compared with surgery performed using an harmonic scalpel. The systemic inflammatory response, however, is significantly affected by numerous factors, including surgery length and the use of drugs to induce and maintain anesthesia (28). Evaluation of the systemic response can vary depending on the time between tissue trauma and the collection of peripheral blood as the half-life of cytokines varies between 6 min for IL-1, $20 \mathrm{~min}$ for TNF and 4-6 h for IL-6 (28). In the present study, qPCR was performed on tissue samples, in order to evaluate the local response that occurs immediately following surgery, hence removing variables connected to time dependence. Our analysis confirmed a significant increase in cytokine expression in tissues cut with a traditional knife, as indicated by the expression of inflammatory mediators in the control samples. The present study demonstrated a wide variation in the expression of genes analyzed, however, statistical analysis verified that only one of the primary mediators of the inflammatory cascade, TNF- $\alpha$, was significantly different among the three tissue samples. Instruments that use electricity or ultrasound energy cause more significant inflammatory protein production than cold knife biopsy. TNF- $\alpha$ is a pleiotropic inflammatory cytokine with local and systemic effects, and is considered a primary mediator responsible for inducing a cascade of cytokines. It increases expression of leukocyte adhesion molecules, stimulates production of IL-1 and chemokines and has procoagulant activity. Additionally, it promotes collagen synthesis and fibroblast proliferation $(29,30)$. Our analysis of protein expression levels in samples obtained by three different surgical devices demonstrated that the new surgical instruments quickly activate the inflammatory cascade. Notably, the present study demonstrated that the ultracision harmonic scalpel set to a higher power level does not cause greater damage than the bipolar forceps. It is reasonable that cytokine production is not significantly different in the two conditions for the given instrumental setting. These results are consistent with those obtained from blood analysis studies by Sietses (27) and Holub (22). However, it should be noted that although qPCR is a highly reproducible technique, laboratory testing may be subject to variations primarily associated with mRNA damage produced by different sampling procedures. If tissue is not immediately frozen the RNA to be extracted may soon become damaged, thus producing samples of poor quality for quantitative analysis. Internal standardization reduces quantitative errors due to RNA degradation.

The evidence that thermal damage is able to induce cell expression of inflammatory response genes led to the hypothesis that the energy use and type of energy used during surgery may affect post-surgical expression of different markers routinely monitored in the postoperative period.

From this hypothesis, it is possible to postulate that the type of energy used during surgery can affect postoperative serum value of certain female genitourinary biomarkers in benign (cancer antigen 125 and anti-mullerian hormones) and malignant diseases (cancer antigen 125 and human epididymis protein 4) (31-33).

Focusing the attention on energy used during surgery in evaluating postsurgical outcomes by serum markers (as usually occur in surgical management of endometriosis) may be useful to solve the majority of discordant results and evidence affected by bias due to different energy used during surgery (34).

In conclusion, in the present study the inflammatory response was analyzed by the quantification of gene expression through the use of qPCR. It was demonstrated that ultracision harmonic scalpel and bipolar forceps trigger the inflammatory cascade and result in an increased production of cytokines compared with cold knife biopsy. RT-qPCR represents a valuable technique to assess tissue damage following surgical trauma through quantitative evaluation of inflammatory response mediators. The results demonstrated that the principal inflammatory mediator was lower in the tissue fragment obtained using cold knife biopsy and no difference was identified in the activation of the inflammatory cascade between the harmonic scalpel and bipolar forceps. It remains to be elucidated whether the observed variability may become significant at different instrument settings, in particular with ultrasound device power 3 . Further studies are required to investigate whether these alterations in the local inflammatory response affect postoperative morbidity.

\section{Acknowledgements}

The authors would like to thank the Gynecological Unit and Pharmacological Department of the University of Padua for their assistance in specimen collection and processing.

\section{References}

1. Harrell AG, Kercher KW and Heniford BT: Energy sources in laparoscopy. Semin Laparosc Surg 11: 201-209, 2004.

2. Advincula AP and Wang K: The evolutionary state of electrosurgery: where are we now? Curr Opin Obstet Gynecol 20: 353-358, 2008.

3. Gizzo S, Burul G, Di Gangi S, Lamparelli L, Saccardi C, Nardelli GB and D'Antona D: LigaSure vessel sealing system in vaginal hysterectomy: safety, efficacy and limitations. Arch Gynecol Obstet 288: 1067-1074, 2013.

4. Sutton PA, Award S, Perkins AC and Lobo DN: Comparison of lateral thermal spread using monopolar and bipolar diathermy, the Harmonic Scalpel and the Ligasure. Br J Surg 97: 428-433, 2010. 
5. Kunde D and Welch C: Ultracision in gynaecological laparoscopic surgery. J Obstet Gynaecol 23: 347-352, 2003.

6. Wallwiener CW, Rajab T, Krämer B, Isaacson KB, Brucker S and Wallwiener M: Quantifying electrosurgery induced therma effects and damage to human tissue: an exploratory study with the fallopian tube as a novel in-vivo in-situ model. J Minim Invasive Gynecol 17: 70-77, 2010.

7. Campbell PA, Cresswell AB, Frank G and Cuschieri A: Real-time thermography during energized vessel sealing and dissection. Surg Endosc 17: 1640-1645, 2003.

8. Jones CJ, Nardo LG, Litta P and Fazleabas AT: Ultrastructure of ectopic peritoneal lesions from women with endometriosis, including observations on the contribution of coelomic mesothelium. Reprod Sci 16: 43-55, 2009.

9. Saccardi C, Gizzo S, Ludwig K, Guido M, Scarton M, Gangemi M, et al: Endometrial polyps in women affected by levothyroxine-treated hypothyroidism - histological features, immunohistochemica findings and possible explanation of etiopathogenic mechanism: a pilot study. Biomed Res Int 2013: 503419, 2013.

10. Gizzo S, Di Gangi S, Bertocco A, Noventa M, Fagherazzi S, Ancona E, et al: Levonorgestrel intrauterine system in adjuvant tamoxifen treatment: balance of breast risks and endometrial benefits - systematic review of literature. Reprod Sci 21: 423-431, 2014.

11. Franchi L, Patrelli TS, Berretta R, Rolla M, Gizzo S, Gramellini D, et al: Role of D-dimer testing in severe pelvic inflammatory disease: a new usable marker to assess the need for fertility-impairing surgery? Fertil Steril 94: 2372-2375, 2010.

12. Litta P, Saccardi C, Conte L and Florio P: Reverse hysterectomy: another technique for performing a laparoscopic hysterectomy. J Minim Invasive Gynecol 20: 631-636, 2013.

13. Rasmussen R: Quantification on the LightCycler. In: Rapid Cycle Real-time PCR, Methods and Applications. Meuer S, Wittwer C and Nakagawara K (eds). Springer Press, Heidelberg, pp21-34, 2001.

14. Livak KJ and Schmittgen TD: Analysis of relative gene expression data using real-time quantitative PCR and the 2(-Delta Delta C(T)) method. Methods 25: 402-408, 2001.

15. Matthews B, Nalysnyk L, Estok R, Fahrbach K, Banel D, Linz H, et al: Ultrasonic and nonultrasonic instrumentation: a systematic review and meta-analysis. Arch Surg 143: 592-600, 2008

16. Litta P,Fantinato S, Calonaci F, Cosmi E, Filippeschi M,Zerbetto I, et al: A randomized controlled study comparing harmonic versus electrosurgery in laparoscopic myomectomy. Fertil Steril 94: $1882-1886,2010$

17. Holub Z, Jabor A, Kliment L, Lukac J and Voracek J: Laparoscopic lymph node dissection using ultrasonically activated shears: comparison with electrosurgery. J Laparoendosc Adv Surg Tech 12: 175-180, 2002.

18. Noventa M,AnconaE,Saccardi C,Litta P,D'Antona D, Nardelli GB, et al: Could HPV-DNA test solve the dilemma about sentinel node frozen section accuracy in early stage cervical cancer? Hypothesis and rationale. Cancer Invest 32: 206-207, 2014.

19. Berretta R, Patrelli TS, Migliavacca C, Rolla M, Franchi L, Monica M, et al: Assessment of tumor size as a useful marker for the surgical staging of endometrial cancer. Oncol Rep 31: $2407-2412,2014$
20. Phillips CK, Hruby GW, Durak E, Lehman DS, Humphrey PA, Mansukhani MM, et al: Tissue response to surgical energy devices. Urology 71: 744-748, 2008.

21. Perko Z, Pogorelić Z, Bilan K, Tomić S, Vilović K, Krnić D, et al: Lateral thermal damage to rat abdominal wall after harmonic scalpel application. Surg Endosc 20: 322-324, 2006.

22. Holub Z, Jabor A, Sprongl L, Kliment L, Fischlová D and Urbánek S: Inflammatory response and tissue trauma in laparoscopic hysterectomy: comparison of electrosurgery and harmonic scalpel. Clin Exp Obstet Gynecol 29: 105-109, 2002.

23. Jacobi CA, Wenger F, Opitz I and Müller JM: Immunologic changes during minimally invasive surgery. Dig Surg 19: 459-463, 2002

24. Härkki-Sirén P, Sjöberg J, Toivonen J and Tiitinen A: Clinical outcome and tissue trauma after laparoscopic and abdominal hysterectomy: a randomized controlled study. Acta Obstet Gynecol Scand 79: 866-871, 2000.

25. Sietses C, Beelen RH, Meijer S and Cuesta MA: Immunological consequences of laparoscopic surgery, speculations on the cause and clinical implications. Langenbecks Arch Surg 384: 250-258, 1999.

26. Kalff JC, Türler A, Schwarz NT, Schraut WH, Lee KK, Tweardy DJ, et al: Intra-abdominal activation of local infiammatory response within the human muscolaris externa during laparotomy. Ann Surg 237: 301-315, 2003.

27. Sietses C, Eijsbouts QA, von Blomberg BM and Cuesta MA: Ultrasonic energy vs monopolar electrosurgery in laparoscopic cholecystectomy: influence on the postoperative systemic immune response. Surg Endosc 15: 69-71, 2001.

28. Lin E, Calvano SE and Lowry SF: Inflammatory cytokines and cell response in surgery. Surgery 127: 117-126, 2000.

29. Strieter RM, Kunkel SL and Bone RC: Role of tumor necrosis factor-alpha in disease states and inflammation. Crit Care Med 21: S447-S463, 1993.

30. Amiot F, Fitting C, Tracey KJ, Cavaillon JM and Dautry F: Lipopolysaccharide-induced cytokine cascade and lethality in LT alpha/TNF alpha-deficient mice. Mol Med 3: 864-875, 1997.

31. Litta P, D'Agostino G, Conte L, Saccardi C, Cela V, Angioni S, et al: Anti-Müllerian hormone trend after laparoscopic surgery in women with ovarian endometrioma. Gynecol Endocrinol 29: 452-454, 2013.

32. Gizzo S, Ancona E, Saccardi C, D'Antona D, Nardelli GB and Plebani M: Could kidney glomerular filtration impairment represent the 'Achilles heel' of HE4 serum marker? A possible further implication. Clin Chem Lab Med 52: e45-e46, 2014.

33. Patrelli TS, Berretta R, Gizzo S, Pezzuto A, Franchi L, Lukanovic A, Nardelli GB and Modena AB: CA 125 serum values in surgically treated endometriosis patients and its relationships with anatomic sites of endometriosis and pregnancy rate. Fertil Steril 95: 393-396, 2011.

34. Sugita A, Iwase A, Goto M, Nakahara T, Nakamura T, Kondo M, et al: One-year follow-up of serum antimüllerian hormone levels in patients with cystectomy: are different sequential changes due to different mechanisms causing damage to the ovarian reserve? Fertil Steril 100: 516-522, 2013. 